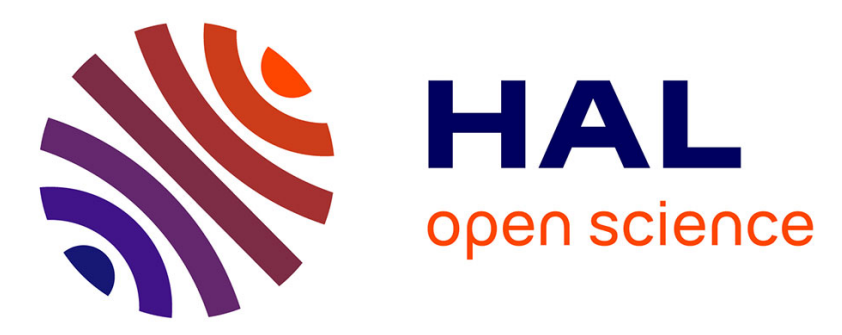

\title{
Contribution à l'étude du champ électrostatique dans les thermoélectrets de polyéthylène dopés
}

\author{
M. Latour, G. Donnet
}

\section{To cite this version:}

M. Latour, G. Donnet. Contribution à l'étude du champ électrostatique dans les thermoélectrets de polyéthylène dopés. Journal de Physique Lettres, 1976, 37 (6), pp.145-148. 10.1051/jphyslet:01976003706014500 . jpa-00231260

HAL Id: jpa-00231260 https://hal.science/jpa-00231260

Submitted on 1 Jan 1976

HAL is a multi-disciplinary open access archive for the deposit and dissemination of scientific research documents, whether they are published or not. The documents may come from teaching and research institutions in France or abroad, or from public or private research centers.
L'archive ouverte pluridisciplinaire HAL, est destinée au dépôt et à la diffusion de documents scientifiques de niveau recherche, publiés ou non, émanant des établissements d'enseignement et de recherche français ou étrangers, des laboratoires publics ou privés. 


\author{
Classification \\ Physics Abstracts \\ $2.220-8.324-8.822-8.750$
}

\title{
CONTRIBUTION A L'ÉTUDE DU CHAMP ÉLECTROSTATIQUE DANS LES THERMOÉLECTRETS DE POLYÉTHYLĖNE DOPÉS
}

\author{
M. LATOUR et G. DONNET \\ Laboratoire de Physique Moléculaire et Cristalline \\ Centre de Recherches des phases condensées (*) \\ Université des,Sciences et Techniques du Languedoc, 34060 Montpellier, France
}

(Reçu le 27 février 1976, révisé et accepté le 23 mars 1976)

\begin{abstract}
Résumé. - Cette étude définit une technique nouvelle qui par des mesures spectroscopiques s'est révélée assez sensible pour noter les variations locales du champ électrostatique interne d'un diélectrique; les mesures sont relatives à une modification du diélectrique, soit instantanée (méthode dynamique), soit permanente (méthode statique). Pour les thermoélectrets de polyéthylène dopés, les variations locales du champ, dues à des phénomènes de charge d'espace, sembleraient agir sur le degré de cristallinité du polymère.
\end{abstract}

Abstract. - The present work describes a new technique for measuring the electrostatic field in a dielectric. The sensibility of this method is such that one can detect local distortions of the field. These measures are relative to instantaneous and permanent modifications of the dielectric (dynamic and static methods). In the case of doped polyethylene thermoelectrets, the local variations of the electrostatic field, due to space charge phenomena, would act on the degree of crystallinity of the polymer.

1. Matériel et méthodes. -1.1 Le MATÉRIEL. Le polyéthylène ( $\mathrm{PE})$ employé dans cette étude a été fourni par la société ATO Chimie [1] avec la référence $1002 \mathrm{CN} 22$.

Les échantillons se présentent sous forme de plaque de 1 à $4 \mathrm{~mm}$ d'épaisseur, dopés par $\mathrm{NaCl}$ à un taux de $500 \mathrm{ppm}$. Leur densité est de $0,920 \mathrm{~g} / \mathrm{cc}$ à $23^{\circ} \mathrm{C}$, la cristallinité est voisine de $49 \%$.

Le spectrographe utilisé pour l'étude Raman est un appareil CODERG PH 1 utilisant comme source un laser rouge Hélium-Néon (6 $328 \AA$ ); $P=600 \mathrm{~mW}$. Les cellules de formation des électrets sont constituées pour permettre la fabrication classique des thermoélectrets. Une cellule spéciale a été conçue pour s'adapter sur le spectrographe afin de suivre l'évolution des spectres au cours de la polarisation du diélectrique. Cette cellule permet d'effectuer des mesures sous vide et de déplacer l'échantillon dans les trois directions horizontales et verticale.

1.2 LES MÉTHODES. - 1.2.1 La méthode de formation des thermoélectrets a déjà été précisée dans des travaux précédents [2]. Dans le cas du PE la température maximum est de $90^{\circ} \mathrm{C}$, l'intensité du champ polarisant varie suivant l'épaisseur de l'échantillon

(*) L.A. 233 entre 16 et $500 \mathrm{kV} / \mathrm{cm}$, avec un maximum de $250 \mathrm{kV} / \mathrm{cm}$ pour les échantillons les plus épais.

1.2.2 Les mesures spectroscopiques sont effectuées par la méthode conventionnelle de dispersion à angle droit en lumière polarisée, entre 1000 et $1500 \mathrm{~cm}^{-1}$. La précision de cette méthode est basée en grande partie sur la sensibilité et la finesse d'enregistrement des spectres Raman. On se référera en particulier aux mesures classiques de hauteur des bandes, largeur à mi-hauteur, et surface. Les mesures étant effectuées entre 1000 et $1500 \mathrm{~cm}^{-1}$ la hauteur des bandes a été normalisée à 1 pour la vibration $v=1296 \mathrm{~cm}^{-1}$, la plus intense dans cette partie du spectre. La limite de détection est fixée à $0,05 \%$. La comparaison de l'intensité de deux bandes Raman, nécessite d'observer rigoureusement la symétrie de ces bandes; en particulier, il est absolument nécessaire de faire toutes les mesures en lumière polarisée.

Deux types d'expérience correspondant à deux méthodes différentes ont été réalisés :

1.2.3 Méthode statique. - Les échantillons sont polarisés préalablement par la méthode classique de formation des thermoélectrets, les électrodes constituées par un dépôt de carbone colloïdal peuvent être retirées afin de permettre l'observation optique de l'échantillon. L'étude est basée sur la comparaison des spectres obtenus pour l'électret et pour un témoin 
ayant subi le même traitement thermique sans être soumis au champ polarisant [3]. Nous avons employé cette méthode pour étudier la répartition du champ en différents points de la tranche de l'électret, comme il est indiqué sur le schéma de la figure 1. En déplaçant verticalement l'échantillon, il est possible de comparer les spectres obtenus pour des positions différentes de l'électret, et d'en déduire grâce à un étalonnage préalable, la répartition relative correspondante du champ en chaque point de la tranche de l'électret.

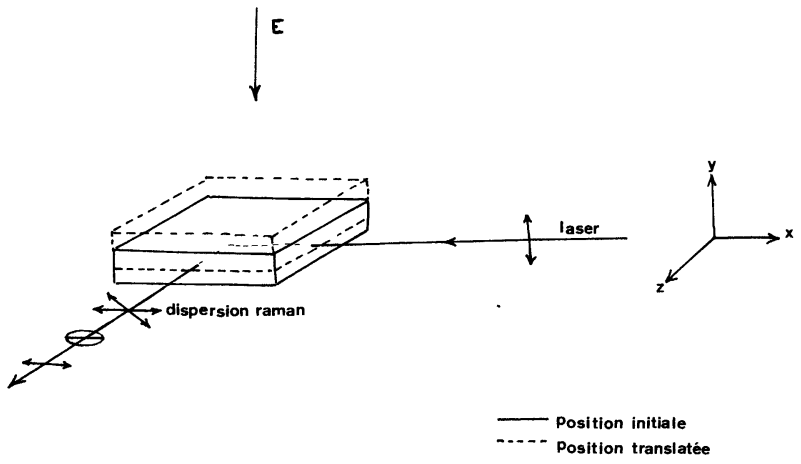

Fig. 1. - Schéma du principe des mesures par spectroscopie Raman.

Remarque. - La structure du polymère n'étant pas homogène, il est nécessaire de réaliser une série de mesures identiques dans un même plan horizontal. L'échantillon observé est taillé dans une région soumise au cours de la polarisation, à un champ électrostatique uniforme, non influencé par les effets de bord.

1.2.4 Méthode dynamique. - Par cette méthode les échantillons sont observés par spectroscopie directement au cours de la polarisation. On note pour une position fixée du diélectrique l'évolution du spectre correspondant, lors de l'application d'un gradient de température ou du champ polarisant. Le balayage que nous avons choisi entre 1000 et $1500 \mathrm{~cm}^{-1}$ durant environ $10 \mathrm{~min}$., ceci nous donne la fréquence des pointés, elle peut évidemment être accélérée si l'on ne considère que l'évolution d'une seule bande caractéristique.

Remarques. - Cette deuxième méthode a sur la précédente l'avantage de ne pas être liée aux défauts de structure ou d'homogénéité du polymère, elle rend compte de l'évolution d'un domaine très précis du diélectrique lorsque celui-ci est soumis à différents paramètres : température, champ électrostatique, polarité du champ, etc... L'enregistrement des spectres par cette méthode est également beaucoup plus facile que par la première méthode nécessitant un réglage très minutieux de la position de l'échantillon avant chaque mesure. Nous avons, par la méthode dynamique, procédé à l'étalonnage des spectres en fonction de l'intensité du champ polarisant : cette méthode nous a également permis de connaître la précision de la méthode statique, nous avons constaté, que les valeurs moyennes du champ déduit ne diffèrent que de 4 à $5 \%$ de la valeur supposée exacte trouvée par la méthode dynamique.

2. Résultats. - L'attribution des bandes correspondant aux différents modes de vibrations du PE a été étudiée par de nombreux auteurs en particulier par Krimm [4], Schachtschneider [5], Gall, Hendra et al. [6]. Le tableau I indique à titre de rappel l'attribution de ces bandes entre 1000 et $1500 \mathrm{~cm}^{-1}$.

\section{TABLEAU I}

Attribution des bandes de vibrations du polyéthylène entre 1000 et $1500 \mathrm{~cm}^{-1}$

\begin{tabular}{|c|c|}
\hline $\begin{array}{l}\text { Fréquences observées } \\
\qquad \mathrm{cm}^{-1}\end{array}$ & Attribution [7] \\
\hline 一 & - \\
\hline 1063 & vas $(\mathrm{C}-\mathrm{C})$ Squel. \\
\hline 1080 & \\
\hline 1131 & $v s(\mathrm{C}-\mathrm{C})$ Squel. \\
\hline 1170 & $\rho r\left(\mathrm{CH}_{2}\right)$ \\
\hline 1280 & \\
\hline 1296 & $\rho t\left(\mathrm{CH}_{2}\right)$ \\
\hline 1306 & \\
\hline 1370 & $\delta s\left(\mathrm{CH}_{3}\right)$ \\
\hline 1418 & $\rho w\left(\mathrm{CH}_{2}\right)$ \\
\hline 1440 & $\delta s\left(\mathrm{CH}_{2}\right)$ \\
\hline 1461 & $\delta\left(\mathrm{CH}_{2}\right)$ \\
\hline
\end{tabular}

2.1 PAR LA MÉTHOde DYNAMIQUE. - Nous avons réalisé l'étalonnage des spectres en fonction de l'intensité du champ polarisant. La figure 2 indique en lumière polarisée perpendiculairement l'évolution des spectres obtenus pour des électrets polarisés sous des champs de 200 à $470 \mathrm{kV} / \mathrm{cm}$, avec en référence, le spectre de l'échantillon non polarisé mais soumis au même gradient thermique.

On constate des modifications assez importantes de l'intensité de certaines bandes : les trois bandes principales qui apparaissent entre 1400 et $1500 \mathrm{~cm}^{-1}$ ( 1418,1440 et 1461 ) présentent, dans le cas de l'électret, un affinement très net de leur configuration en particulier leur largeur à mi-hauteur $\left(\mathrm{en}^{\mathrm{cm}}{ }^{-1}\right)$ est nettement réduite, leur hauteur varie également légèrement. Le tableau II donne les valeurs de l'intensité relative (hauteur des pics), la largeur à mi-hauteur et la surface de ces trois bandes pour des électrets formés sous 200 et $470 \mathrm{kV} / \mathrm{cm}$ ainsi que pour le témoin.

On constate également figure 2 , que dans le cas de l'électret, les bandes 1080 et $1306 \mathrm{~cm}^{-1}$ ont une intensité relative plus faible, leur hauteur décroissante en fonction inverse de l'intensité du champ polarisant.

Ces différentes variations laisseraient supposer une modification du degré de cristallinité des échantillons. Les travaux de Gall, Hendra, Peacock, Cudby et Willis [7] relatifs à la spectroscopie Raman d'échan- 


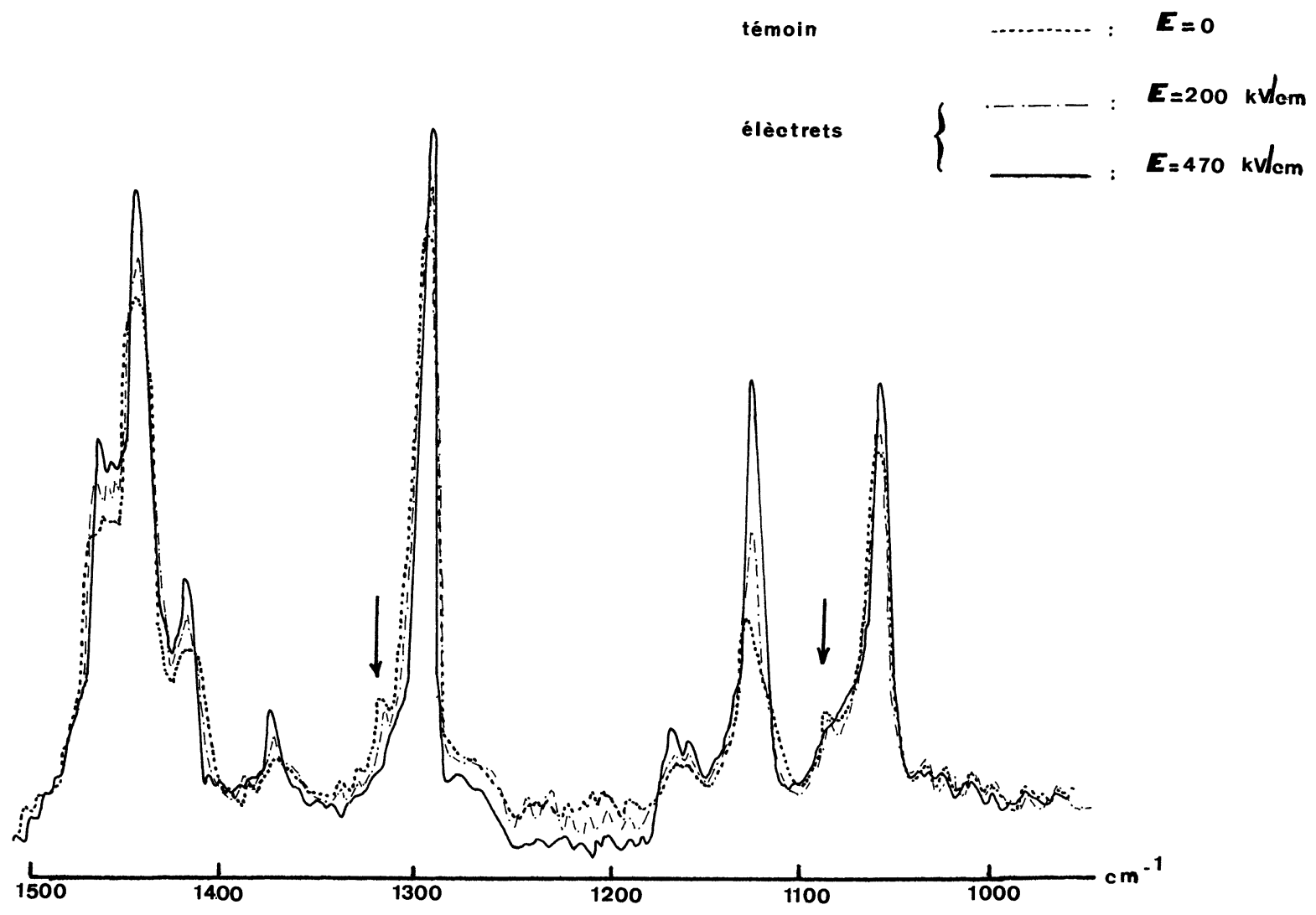

Fig. 2. - Evolution du spectre du PE entre 1000 et $1500 \mathrm{~cm}^{-1}$ en fonction de l'intensité du champ polarisant.

tillons de PE de degré de cristallinité différents ont révélé des modifications spectrales voisines de nos résultats en ce qui concerne le domaine de fréquence compris entre 1000 et $1500 \mathrm{~cm}^{-1}$; ceci indiquerait, comme nous l'avions déjà précisé précédemment pour les électrets de $\mathrm{PVF}_{2}$ [3], [8], que l'action du champ polarisant associée à l'effet de température tel qu'il intervient dans la formation des thermoélectrets provoquerait une légère augmentation du degré de cristallinité du polymère.

\section{TABLEAU II}

Variation de la largeur à mi-hauteur, de la hauteur et de la surface des bandes : 1440,1418, $1461 \mathrm{~cm}^{-1}$, en fonction de l'intensité du champ de polarisation

$$
\Delta v=1418 \mathrm{~cm}^{-1}
$$

Témoin

Electret $E=200 \mathrm{kV} / \mathrm{cm}$

Electret $E=470 \mathrm{kV} / \mathrm{cm}$

$\Delta v=1440 \mathrm{~cm}^{-1}$

Témoin

Electret $E=200 \mathrm{kV} / \mathrm{cm}$

Electret $E=470 \mathrm{kV} / \mathrm{cm}$

$\Delta v=1461 \mathrm{~cm}^{-1}$

Témoin

Electret $E=200 \mathrm{kV} / \mathrm{cm}$

Electret $E=470 \mathrm{kV} / \mathrm{cm}$
Intensité relative (hauteur des pics) $\left(^{*}\right)$

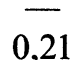

0,28

0,36

0,67

0,80

0,90

0,38

0,54

0,58
Largeur

à mi-hauteur $\left(\mathrm{cm}^{-1}\right)$

$\overline{16,2}$

15,0

12,5

17,5

16,2

15,0

22,5

21,5

20
Surface des pics

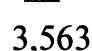

4,230

4,515

11,85

13,05

13,54

11,650

11,612

(*) Les hauteurs sont normalisées à 1 sur la bande $1296 \mathrm{~cm}^{-1}$. 
Etalonnage des spectres. - L'échantillon de $1 \mathrm{~mm}$ d'épaisseur, placé dans la cellule, est soumis d'abord au gradient thermique puis à des champs polarisants d'intensité variable. Pour chaque valeur du champ, un spectre est enregistré. Les variations de surface $\Delta S$ des bandes 1440 et $1418 \mathrm{~cm}^{-1}$, correspondant respectivement aux vibrations $\delta s\left(\mathrm{CH}_{2}\right)$ et $\rho w\left(\mathrm{CH}_{2}\right)$, sont indiquées figure 3 . $\Delta S$ représente la différence de surface des bandes entre l'échantillon polarisé et non polarisé. Cet étalonnage est relatif aux variations spectrales d'une zone bien localisée et fixe du diélectrique, en fonction de l'intensité du champ polarisant appliqué à l'échantillon. On suppose que dans cette zone définie par la largeur du faisceau laser et le champ interne est uniforme, s'il ne l'est pas nous enregistrons une valeur moyenne des perturbations locales en ce point. La précision de l'étalonnage est liée à la finesse du faisceau laser et à la précision de l'enregistrement.

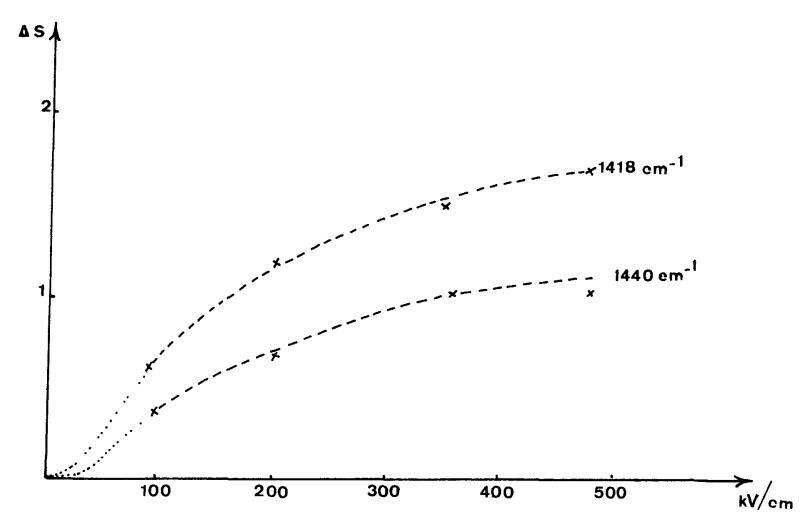

FIG. 3. - Variations de la surface des bandes 1440 et $1418 \mathrm{~cm}^{-1}$ en fonction de l'intensité du champ polarisant.

2.2 PAR LA MÉTHOde STATiQue. - Nous avons relevé les valeurs de l'intensité relative du champ électrostatique pour sept positions différentes du diélectrique distantes chacune de $0,25 \mathrm{~mm}$. Chaque position est obtenue par des déplacements successifs de l'échantillon sur une même verticale. La bande $1440 \mathrm{~cm}^{-1}$ a été prise comme référence. Pour chaque position de l'échantillon on note la variation de surface de la bande. Grâce à l'étalonnage précédent nous en déduisons la valeur correspondante du champ relatif.

La figure 4 donne le schéma de la répartition relative du champ à l'intérieur du diélectrique suivant un plan perpendiculaire à la surface des électrodes. Les électrets sont polarisés sous un champ de $160 \mathrm{kV} / \mathrm{cm}$. La courbe $(b)$ correspond au cas des électrodes bloquantes, la courbe $(a)$ au cas des électrodes métalliques.

D'après la courbe $(b)$ on peut supposer que le champ serait dû, apparemment, à un même type de charge (hétérocharge), plus faible au centre que sur les bords au voisinage des électrodes, avec un maximum du côté de l'anode, ce qui peut s'expliquer par des différences de mobilité entre les électrons et les porteurs de charges positifs. La courbe $(a)$ montre que dans le

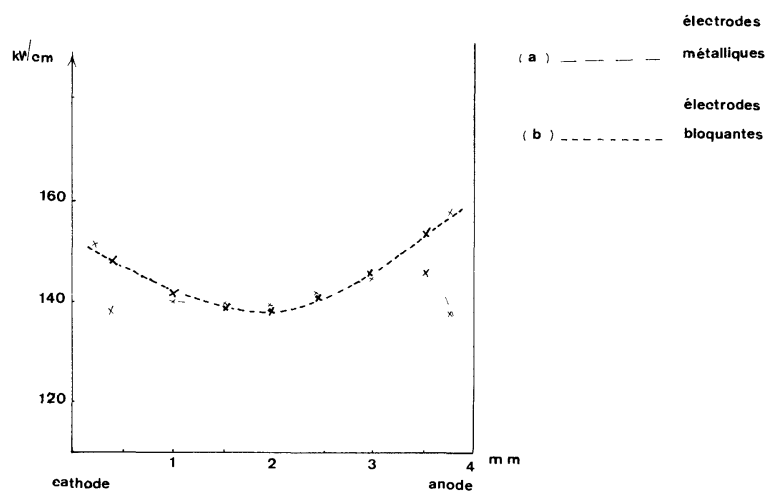

FIG. 4. - Répartition relative du champ interne du diélectrique polarisé sous une tension de $+160 \mathrm{kV} / \mathrm{cm}$. a) Electrodes bloquantes; $b$ ) Electrodes injectantes.

cas d'électrodes métalliques, il y a au voisinage des électrodes une distorsion du champ local qui pourrait être dû à l'injection d'un deuxième type de charge (homocharge) de signe opposé à celles observées dans le cas précédent ; la répartition du champ au centre de l'électret semble analogue à celle obtenue sur la courbe $(b)$, l'homocharge n'affecterait que la partie externe du diélectrique.

Cette interprétation des résultats, serait en accord avec les travaux de Perret [9].

\section{Bibliographie}

[1] Echantillons fournis dans le cadre de la RCP 370 sur l'étude des charges d'espace dans le polyéthylène.

[2] Latour, M., J. Appl. Phys. 46 (1975) 1.

[3] Latour, M., International meeting on electrets and dielectrics Sao Carlos (août 1975). A paraître dans J. Acc. Sc. Braz. (1976).

[4] KRIMS, S., Fortschr. Hochpolym. Forsch. 2 (1960) 51.

[5] Schachtschneider, H. J., SNyder, R. G., Spectrochim. Acta 19 (1963) 117.
[6] SNyder, R. G., J. Macromol. Spectroscopy 37 (1971) 2

[7] Gall, M. J., Hendra, P. J., Peacock, C. J., Cudby, M. E. A., WILlIS, H. A., (a) Spectrochim. Acta 28 A (1972) 1485.

Gall, M. J. Hendra, P. J., Peacock, C. J., Cudby, M. E. A., Willis, H. A., (b) Polymer 13 (1972) 3.

[8] Latour, M., A paraître IVe Congrès Européen de spectroscopie des hauts polymères. Strasbourg, mars (22-24) 1976.

[9] Perret, J., Bull. E.D.F., D.E.R., série B, no 2 (1974) 22̇-70. 